\title{
CASA DE PUERTO RICO EN ESPAÑA: ORIGEN YTRAYECTORIA DESDE MEDIADOS DEL SIGLO XX
}

\author{
House of Puerto Rico in Spain: origin and trajectory \\ since the mid-20 $2{ }^{\text {th }}$ century
}

\author{
Rafael Pérez Colón \\ Casa de Puerto Rico en España (España)
}

En el 1898 la relación entre Puerto Rico y España entra en etapa de transición al terminar los más de cuatrocientos años de soberanía española en la isla. Bajo la bandera de los Estados Unidos de América, Puerto Rico comienza una nueva andadura en lo político, económico, social y cultural a principios del siglo XX. Paralelamente, España entra en una etapa de inestabilidad política que culmina en una guerra civil. Sin embargo, los vínculos forjados a lo largo de cuatrocientos años de historia común logran mantener viva la conexión entre familias y otras actividades sobre la base de una lengua común y cultura afín. Más de un siglo después de iniciada esta transición, Puerto Rico sigue caracterizándose por su lengua y cultura hispánica. Fueron muchas las instituciones y personas que contribuyeron a nutrir el hispanismo en la isla y la relación con España a lo largo de este período. En este contexto se crea la Casa de Puerto Rico en España (CAPRE), formalmente en 1965, en Madrid y a partir de organizaciones de estudiantes puertorriqueños en universidades españolas. CAPRE surge del interés de la comunidad de puertorriqueños establecida en España por dar a conocer la isla y lo que allí pasaba, a la vez que contribuían a fortalecer el histórico vínculo. El Gobierno de Puerto Rico también tenía interés en dar a conocer los progresos de la isla y apoyó la iniciativa desde sus inicios. Hasta la apertura en Madrid de una oficina del Gobierno de Puerto Rico en la década de los ochenta, CAPRE fue uno de los más activos actores y vínculo en la promoción de las relaciones entre la isla y España. Desde su fundación hasta la actualidad, CAPRE mantiene su actividad enfocada en dar presencia de Puerto Rico en la sociedad española y espera seguir haciéndolo en el futuro.

\section{Palabras clave}

Puerto Rico, CAPRE, cultura puertorriqueña, Ramón-Darío Molinary, Instituto de Cultura Hispánica, Casa de América, exilio español en Puerto Rico, Seminario de Cultura Puertorriqueña, Rafael Pérez Colón
In 1898 the relationship between Puerto Rico and Spain entered a transition phase after more than 400 years of Spanish sovereignty on the island. Under the banner of the United States of America, Puerto Rico began a new journey in the political, economic, social and cultural aspects within the early twentieth century. At the same time, Spain enters a stage of political instability that culminates in a civil war. However, the bonds forged over 400 years of common history manage to keep alive the connection between families and other activities based on a common language and cultural ties. More than a century after this transition began, Puerto Rico continues to be characterized by its Spanish language and Hispanic culture heritage. There were many institutions and people who contributed to nurturing the Hispanic ties on the island and the relationship with Spain throughout this period. In this context the House of Puerto Rico in Spain (CAPRE) was created, formally in 1965, in Madrid and as a continuation of Puerto Rican student organizations in Spanish universities. CAPRE arises from the interest of the Puerto Rican community established in Spain, to publicize the island and its developments while helping to strengthen the historic link. The government of Puerto Rico was also interested in publicizing the island's progress and supported the initiative from its inception. Until the opening in Madrid, of an office of the government of Puerto Rico, in the 1980s, CAPRE was one of the most active actors and liaison in the promotion of relations between the island and Spain. From its foundation, to the present day CAPRE maintains its activity focused on giving presence to Puerto Rico within the Spanish society and looks forward to staying on.

\section{Keywords}

Puerto Rico, CAPRE, Puerto Rican culture, Ramón-Darío Molinary, Institute of Hispanic Culture, Casa de América, Spanish exile in Puerto Rico, Seminar of Puerto Rican Culture, Rafael Pérez Colón 

Puerto Rico y España entró en una etaa de transición que se inició con la invasión de la isla por parte de los Estados Unidos de América y la firma del Tratado de París de 1898 que convirtio a Puerto Rico en propiedad de la meras décadas del siglo XX fueron de gran complejidad a nivel global. Entre los múltiples desafíos enfrentados por nuestra sociedad, valga mencion que pasamos por dos guerras mundiales, por un período de inestabilidad política y guerra civil en España y por la depresión económica de los años treinta en los Estado Unidos.

Durante este convulso período, en Puerto Rico, la imposición del nuevo modelo colonial que se inició en 1898 ponía a prueba una cultura y sociedad construidas a lo largo de cuatrocientos años de vida bajo la bandera española. A dicha cultura y sociedad, forjadas a partir de la fusión entre taínos, europeos (principalmente españoles) y africanos, se impuso la influencia cultural de la nueva metrópoli. Al mismo tiempo, la vuelta a la condición colonial de la isla representó un retroceso en su desarrollo político. Este retroceso implicó el inicio de un proceso de descolonización que un siglo más tarde sigue inconcluso.

La segunda mitad del siglo XX fue fructífera tanto para Puerto Rico como para España en materia de desarrollo económico y modernización. Este período será determinante para la creación de un nuevo modelo de relación entre ambos países, basado en su historia e intereses comunes y no en la relación política. Entrados en el siglo XXI, luego de más de un siglo bajo la bandera de los Estados Unidos, Puerto Rico sigue manteniendo el español como su lengua y el hispanismo como elemento clave de su cultura e identidad. Asimismo, el intercambio cultural, social, académico, cientifico y comercial se ha convertido en una constante de la relación entre Puerto Rico y España, proyectándose hacia el futuro. Cabe destacar que, a partir del vínculo histórico-cultural, fueron muchos los factores y actores que incidieron, tanto en la isla como en España, para la creación de mecanismos que permitieran cultivar y fortalecer la nueva relación entre ambos pueblos a lo largo del último siglo. Uno de estos factores fue la emigración y exilio de españoles que, durante la guerra civil española y la dictadura, fueron recibidos en Puerto Rico y contribuyeron en gran medida a reforzar la hispanidad en la sociedad puertorriqueña

Tanto la guerra hispano-estadounidense como la invasión y
transición de Puerto Rico han sido ampliamente estudiadas a lo largo de los años. Una referencia singular fue editada por CAPRE
como libro conmemorativo de su 30 aniversario en 1996 bajo el título Puerto Rico ante el 98 visto desde España. de entonces. Podemos destacar la huella que dejaron en la isla intelectuales como María Zambrano Juan Ramón Jiménez, Pablo Casals, Pedro Salinas monseñor Vicente Murga, entre otros ${ }^{2}$.

Paralelamente, la presencia de los puertorriqueños en España se mantuvo a lo largo del siglo XX anto por vínculos familiares como por el comercio $u$ otros motivos, entre los que destacamos la presencia de jóvenes puertorriqueños que escogieron España como su destino de estudios universitarios. Este último fenómeno destaca a lo largo de varias décadas de mediados del siglo. Es así como, en el se organizarorsitario español, los puertorriqueños y conocimiento sobre la situación de la isla. Cabe mencionar que un porcentaje significativo de estos estudiantes contrajo matrimonio en España y algunos establecieron sus familias en la península, mientras que otros regresaron a la isla con sus nuevas familias y vínculos permanentes con España. Estos nuevos vínculos familiares sirvieron de apoyo y ayudaron a renovar las relaciones a ambos lados de Atlántico.

Sobre las agrupaciones de estudiantes universitarios puertorriqueños constan referencias de grupos como el Círculo Cultural Puertorriqueño de Salamanca, el Círculo Universitario Puertorriqueño de Barcelona, la Sociedad Universitaria Puertorriqueña de Santiago de Compostela o el Círculo Cultural de Estudiantes Puertorriqueños, que estuvo activo en Madrid desde 1949 hasta 1962. En 1959 se estimaba en más de quinientos el numero de estudiantes universitarios puertorriqueños en Madrid, siendo considerada como la mayor comunidad proveniente de Hispanoamérica en el ámbito universitario de la capital española. Igualmente, en universidades como la de Salamanca los estudiantes puertorriqueños constituían una de las principales comunidades extranjeras.

Esta fuerte presencia y activismo de los estudiantes puertorriqueños llamó la atención de monseñor Vicente Murga, quien se convierte en actor clave de lo que pasó a ser un hito en la organización de la comunidad de estudiantes puertorriqueños en España. Monseñor Vicente Murga Sanz (1903-1976) fue un sacerdote español que se estableció en Puerto Rico en 1932 y dejó un importante legado en la isla.

La huella del exilio español en Puerto Rico ha sido ampliamente Vázquez, C. (2011): Breves notas sobre el exilio español en Puerto Rico. Recuperado el 6 de abril de 2020, de hittps://dial/net.unirioja El exilio en Puerto Rico de los artistas españoles de la diáspo ra republicana de 1939. Recuperado el 6 de abril de 2020, de hittps://digital.csic.es/bitstream/10261/75558/1/Puerto\%20Rico
Exilo\%20Artistas 2012.pdf
Cabe destacar su participación en la fundación de la Pontificia Universidad Católica de Puerto Rico su labor como investigador e historiador sobre colonización española en la isla.

En 1959 monseñor Murga se encontraba en EsEaña investigando en los archivos históricos sobre la colonización de la isla cuando entró en contacto con los estudiantes puertorriqueños en Madrid to concibieron la idea pe crear una en Madríd representativa de la comunidad de estudiante puertorriqueños en España para dar mayor difusión a lo que estaba sucediendo en la islayor difusión también fue reconocida por parte del Gobierno de Puerto Rico y de la Embajada de Estados Unidos en España.

Esta idea fue presentada al Instituto de Cultura Hispánica, donde fue bien acogida, materializándose el esfuerzo con la fundación en 1959 de la Cas de Puerto Rico, albergada en dicho instituto. A continuación, incluimos una foto del grupo fundado durante el evento de inauguración en Madrid.

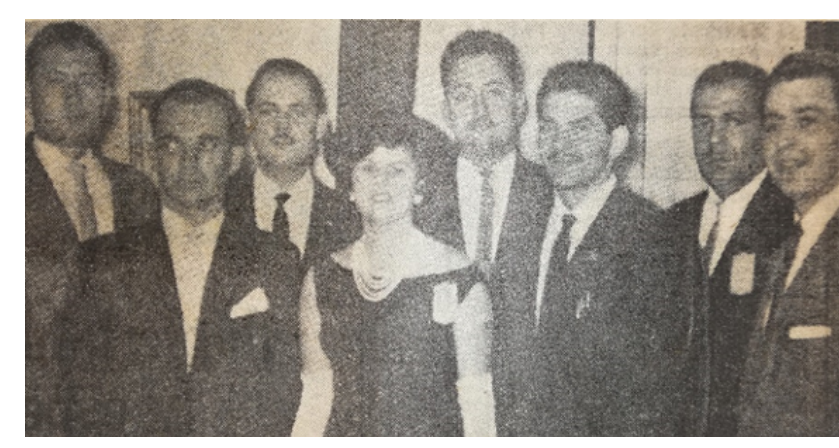

Directiva fundadora de la Casa de Puerto Rico, 1959. De izquierd a derecha: Galileo Ramírez Baera (vocal), Juan Tomasini (presichez (secretaria), Ramón-Dario Molinary (vicepresidente), Rafae Sierra (vocal). Fuente: Periódico El Imparcial, Puerto Rico, edición

Así pasamos de contar con diversos grupos uniespañolas a constituir una organización con sede fisica en Madrid que se proponía como un promotor del conocimiento sobre Puerto Rico ante la socledad española. Cabe destacar que, con la constitución del Estado Libre Asociado de Puerto Rico (1952), tanto el Gobierno de los Estados Unidos como el Gobierno de Puento Rico estaban ávidos de mostrar al mundo los avances de la isla en materia política, económica y social. Claramente, tanto monseñor Murga como los puertorriqueños en España compartian este interés.

Ya para 1965, los avances en el desarrollo económico y social de Puerto Rico eran noticia a nive mundial y el interés por conocer más sobre lo que pasaba en la isla aumentaba en España y al mismo tiempo el interés por darlo a conocer aumentaba por parte del gobierno de la isla. Sin embargo, la capacidad de actuación de la Casa de Puerto Rico se veía limitada por las restricciones propias del tiempo disponible por parte de los estudiantes universitarios para desarrollar y mantener un programa de actividades

Coinciden así una oportunidad y una preocupación que fue recogida por puertorriqueños que se habian establecido en España en aquella épose Algunos de ellos habían sido estudiantes que de las actividades de la Casa de Puerto Rico y ortras iniciativas. Es así como surge la idea de crear una nueva organización liderada por puertorriqueños aueva organizacion liderada por puertorriqueños yor capacidad a los esfuerzos de la comunidad de estudiantes.

Esta combinación de factores sirvió de plataforma para la constitución en Madrid de la Casa de Puerto Rico en España (CAPRE) el 13 de marzo de 1965. La presidencia la ocupó Ramón-Darío Molinary Matías, quien también había sido vicepresidente y cofundador de la Casa de Puerto Rico albergada en el Instituto de Cultura Hispánica durante su época de estudiante. Además de ejercer su profesión como periodista, corresponsal de prensa extranjera en Madrid para varios medios y presidente de la Asociación de Corresponsales de Prensa Extranjera en España durante un largo período, Molinary ocupó la dirección de CAPRE desde su fundación en 1965 hasta su retiro en 2018

CAPRE ha contado con importantes apoyos personales e institucionales desde su fundación. Entre ellos destaca la labor del escritor, economista y académico puertorriqueño don Ramón Piñeiro León como cofundador de CAPRE y distinguido miembro de la comunidad puertorriqueña en España. Entre otros reconocimientos, Piñeiro León recibio el reconocimiento del Ayuntamiento de Madrid con una placa conmemorativa en la dirección donde vivió hasta su muerte.

El momento de la fundación de CAPRE en Madrid coincide con un momento especial en Puerto Rico, donde la llamada Operación Manos a la Obra habia sido motor de un importante crecimiento económico y la isla entraba en la etapa que fue conocida como Operación Serenidad ${ }^{4}$, que ponía

Operación Manos a la Obra fue el nombre dado al conjunto de iniciativas de desarrollo económico que lanzo el Gobierno de la isla en 1947 . Proyecto Salón Hogar (s. f.). Biografía. Operación

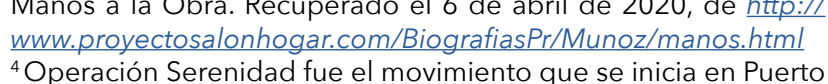
Rico a partir de 1949 estableciendo las instituciones y programas 
prioridad a los esfuerzos en el desarrollo cultural y humano de la isla. En este marco, CAPRE contó con apoyo y colaboración desde el Gobierno de Puerto Rico, particularmente del Instituto de Cultura Puertorriqueña,

Desde su creación, la actividad de CAPRE fue amplia y constante, aunque su primera década de amplia y constante, aunque su primera década de creadas con el interés del Gobierno de la isla y los apoyos recibidos. El legado de la trayectoria de CAPRE hasta la fecha es muy variado y extenso. Del mismo modo lo ha sido el de las asociaciones estudiantiles precursoras de CAPRE. Este legado includiantiles precursoras de CAPRE. Este legado inclua través de la geografía española y en Puerto Rico conciertos musicales, exhibiciones artísticas, publicanciertos musicales, exhibiciones artísticas, publas conmemorativas, concursos y premios sobre ensayos, literatura y otros trabajos sobre Puerto Rico, y una vasta red de colaboradores a nivel personal e institucional. A continuación, algunos ejemplos:

- Placa a don Luis Muñoz Rivera, ubicada en calle Mayor, 13, de Madrid, por Círculo Cultural de Estudiantes Puertorriqueños en 1959.

- Busto de don Eugenio María de Hostos, en el Parque del Oeste de Madrid, por CAPRE en 1980. - Busto de don Ramón Power y Giralt, en la Alameda Apodaca de Cádiz, por CAPRE en 1985. - Placa a don Jose Ramon Pineiro Leon, cofundador de CAPRE, por Ayuntamiento de Madrid.

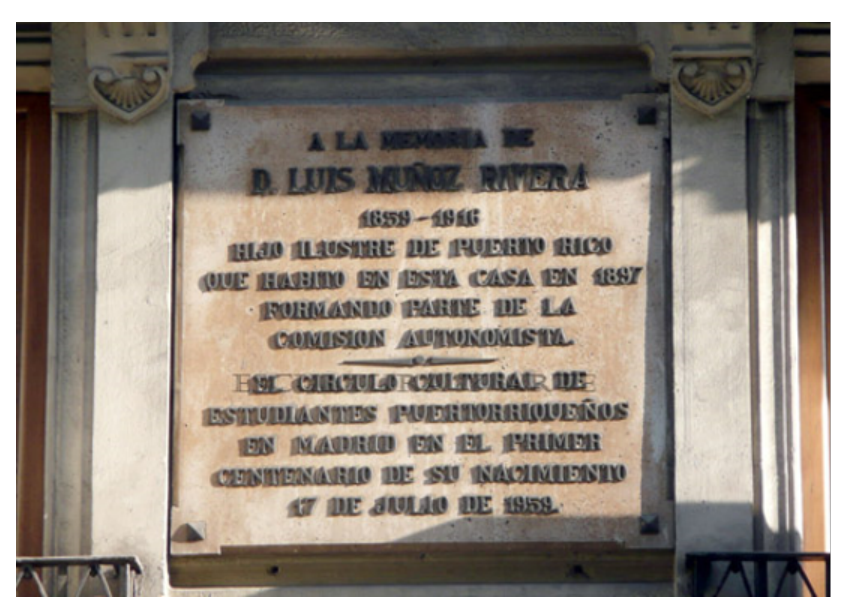

Placa a don Luis Muñoz Rivera en calle Mayor, 13, de Madrid.

que se enfocarán en el desarrollo cultural y social de la población
de la isla. Véase: Proyecto Salón Hogar (s. f.). Biografía. Operrade la isla. Véase: Proyecto Salón Hogar (s. f.). Biografía. Opera-
ción Serenidad. Recuperado el 6 de abril de 2020, de http://www. html

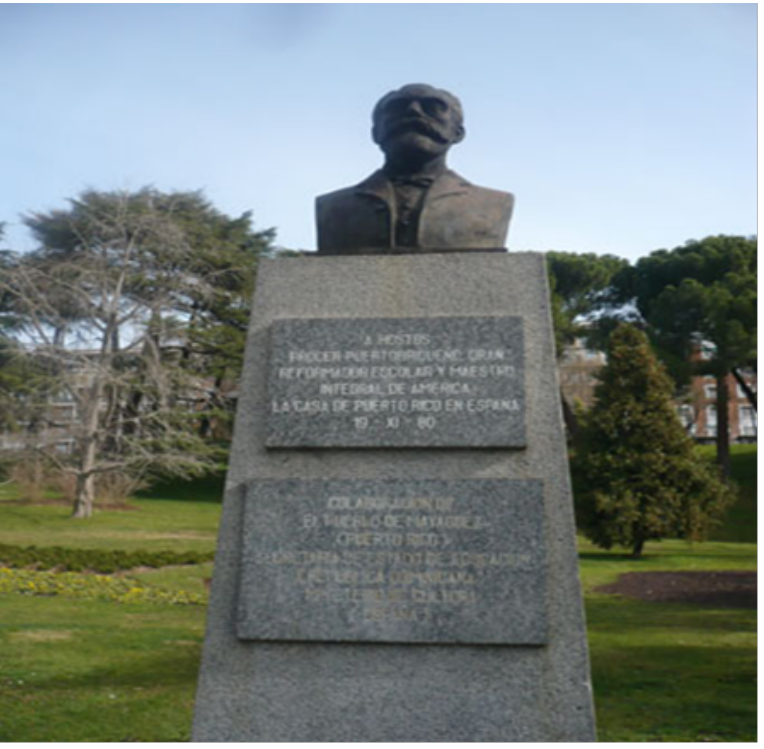

Busto de don Eugenio María de Hostos en Parque del Oeste, en Madrid.

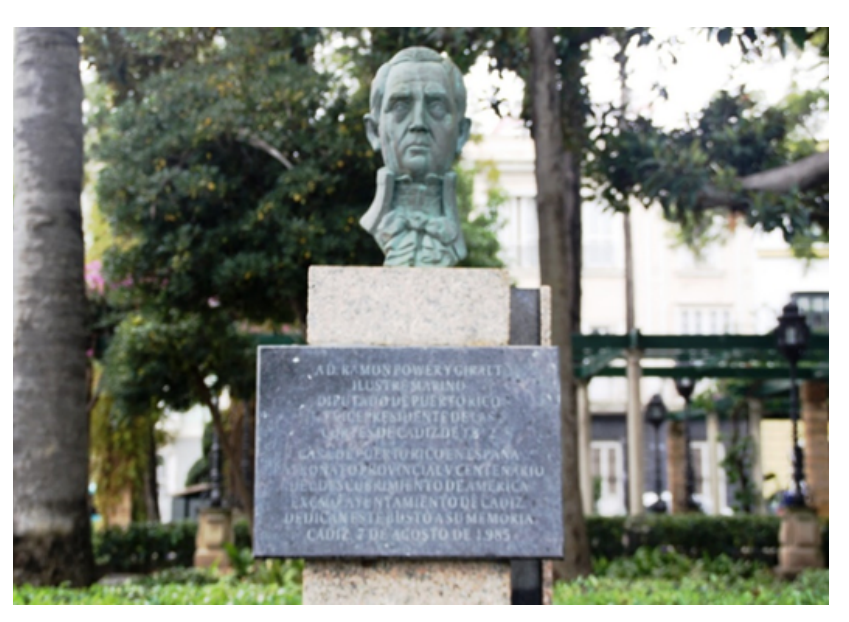

Busto de don Ra
daca, de Cádiz.

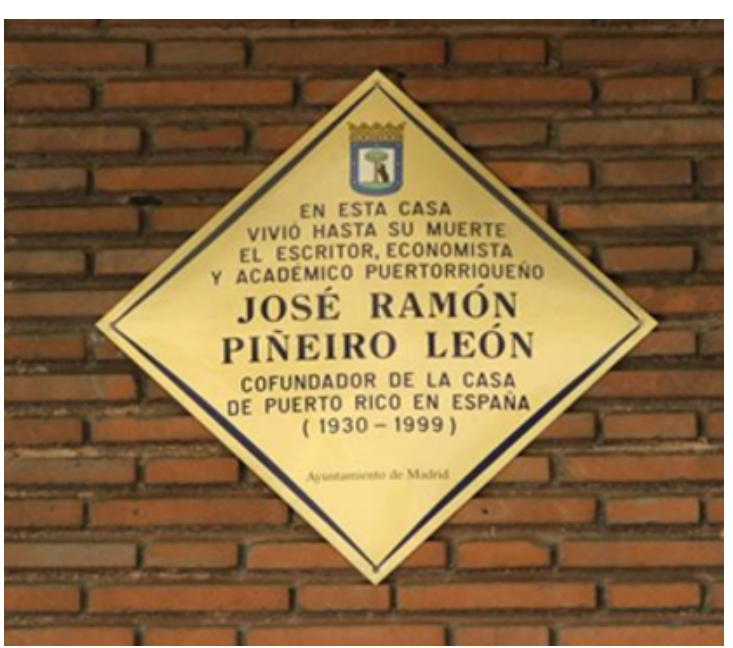

Placa a don José Ramón Piñeiro León, cofundador de CAPRE, en Madrid.
Sin duda, las relaciones entre Puerto Rico y España se transformaron y ganaron en dinamismo a lo largo el siglo XX, acompañadas por la nueva geopolítica global. En la segunda mitad de la década de los chenta un hito importante fue la apertura de una oficina de promoción del Gobierno de Puerto Rico en Madrid. Como era de esperar, la presencia oficial del Gobierno de Puerto Rico en España, con recursos dedicados a la promoción del turismo, desarrollo económico e industrial, y el intercambio académico, agregó fortalezas a la relación.

Otro punto de inflexión importante se da en el período de 1991-1992 con varios acontecimientos de gran visibilidad, entre los que resaltamos los siguientes: la participación de Puerto Rico con un pabellón propio en la Exhibición Universal de Sevilla en 1992 la celebración en Puerto Rico del Quinto Centenario del Descubrimiento de América y Puerto Rico y la obtención del Premio Príncipe de Asturias de las Letras en 1991 por parte del pueblo de Puerto Rico. Durante todo este período CAPRE mantuvo una importante agenda de actividades tanto de forma independiente como en colaboración con la oficina del Gobierno de Puerto Rico, siendo también protagonista durante este ciclo de gran actividad.

El período 2016-2018 marca otro punto de inflexión ante una fuerte crisis económica en Puerto Rico que lleva al cierre de la Oficina del Gobierno de Puerto Rico en Madrid. Coincidentemente, durante esto años llega el retiro de don Ramón-Darío Molinary.

En 2019, Rafael Pérez Colón asume la presidencia de CAPRE y comienza a reactivarse la agenda de actividad a través de la colaboración con la Casa de América y de la Fundación Consejo España-EE. UU. E evento de relanzamiento de CAPRE se celebro el día 10 de octubre con la conferencia Puerto Rico en crisis: ¿qué pasó alli ? $^{5}$, que estuvo a cargo del conocido artista, comunicador y escritor puertorriqueño Silverio Pérez.

La agenda de CAPRE continuó el 25 de noviembre de 2019 con la participación en la mesa redonda

Vídeo del evento de relanzamiento de CAPRE en Casa de pasó alli? Recuperado de hittps://www.youtube.com/watch? $v=$ Aed-7Wn $41 P E$
«Historia y relaciones entre Puerto Rico y España», organizada por el Aula María Zambrano de Estudios Transatlánticos de la Universidad de Málaga, donde se presentó a grandes rasgos este resumen histórico y se esbozó la visión de la nueva etapa. CAPRE se encamina ahora a continuar en el desempeño de su misión, ajustada a las nuevas realidades de Puerto Rico, España y el mundo globalizado, digitalizado y más interdependiente.

La agenda principal mantendrá su prioridad en cultivar el vínculo Puerto Rico-España, promoviendo y dando mayor visibilidad sobre el acontecer culen esta nueva de la isla en la península. Asimismo, desarrollo de oportunidades para mayor colaboración en sectores como el intercambio colabo$c 0$, el I+D, el desarrollo económico y el desarrollo cultural.

Sirva este resumen sobre el origen y trayectoria de CAPRE para reflexionar sobre el papel que ha desempeñado hasta ahora, así como el que podrá desempeñar en el futuro. Sirva también como nota de reconocimiento y agradecimiento a don $\mathrm{Ra}$ món-Darío Molinary por toda una vida de trabajo y dedicación a la promoción y defensa de la cultura e identidad de Puerto Rico y de los puertorriqueños ante la sociedad española. Quizás la mejor forma de expresar este reconocimiento es compartiendo su visión como cierre de este texto con la inclusión de un extracto del discurso que pronunció don Ramón-Darío en el acto de clausura de uno de los primeros eventos organizados por CAPRE, el I Seminario de Cultura Puertorriqueña, que se celebró en Madrid en 1965

No cabe duda, tenemos cultura propia. Y necesitamos conocerla, a fin de desvanecer, con la enseñanza del pasado, las vacilaciones del presente, avanzando con paso mesurado y firme hacia el porvenir. Y necesitamos hacerla conocer a los demás, para que nuestra personalidad regional destaque se nos confunda con nadie, adjudicándosenos solidaridad en faltas que no cometimos, achacándosenos aspiraciones opuestas a nuestra idiosincrasia peculiarísima, suponiéndosenos sentimientos que jamás informaron nuestra vida de paz, de trabajo, de concordia y de fervoroso patriotismo. 